\title{
Modelling the Effects of Immune Response and Time Delay on HIV-1 in Vivo Dynamics in the Presence of Chemotherapy
}

\author{
Cherono Pela ${ }^{1, *}$, Kirui Wesley ${ }^{2}$, Adicka Daniel $^{1}$ \\ ${ }^{1}$ Department of Mathematics and Computer Science, University of Kabianga, Kericho, Kenya \\ ${ }^{2}$ Department of Mathematics and Actuarial Science, South Eastern Kenya University, Kitui, Kenya \\ Email addresses: \\ pelacherono@gmail.com (C. Pela), kirwes@seku.ac.ke (K. Wesley), dadicka@kabianga.ac.ke (A. Daniel) \\ ${ }^{*}$ Corresponding author
}

\section{To cite this article:}

Cherono Pela, Kirui Wesley, Adicka Daniel. Modelling the Effects of Immune Response and Time Delay on HIV-1 in Vivo Dynamics in the Presence of Chemotherapy. Mathematical Modelling and Applications. Vol. 4, No. 2, 2019, pp. 15-21. doi: 10.11648/j.mma.20190402.11

Received: June 10, 2019; Accepted: July 11, 2019; Published: July 22, 2019

\begin{abstract}
Numerous models of mathematics have existed to pronounce the immunological response to contagion by human immunodeficiency virus (HIV-1). The models have been used to envisage the regression of HIV-1 in vitro and in vivo dynamics. Ordinarily the studies have been on the interface of HIV virions, CD4+T-cells and Antiretroviral (ARV). In this study, time delay, chemotherapy and role of CD8+T-cells is considered in the HIV-1 in-vivo dynamics. The delay is used to account for the latent time that elapses between exposure of a host cell to HIV-1 and the production of contagious virus from the host cell. This is the period needed to cause HIV-1 to replicate within the host cell in adequate number to become transmittable. Chemotherapy is by use of combination of Reverse transcriptase inhibitor and Protease inhibitor. CD8+T-cells is innate immune response. The model has six variables: Healthy CD4+T-cells, Sick CD4+T-cells, Infectious virus, Noninfectious virus, used CD8+T-cells and unused CD8+T-cells. Positivity and boundedness of the solutions to the model equations is proved. In addition, Reproduction number $\left(\mathrm{R}_{0}\right)$ is derived from Next Generation Matrix approach. The stability of disease free equilibrium is checked by use of linearization of the model equation. We show that the Disease Free Equilibrium is locally stable if and only if $\mathrm{R}_{0}<1$ and unstable otherwise. Of significance is the effect of CD8+ T- cells, time delay and drug efficacy on stability of Disease Free Equilibrium (DFE). From analytical results it is evident that for all $\tau>0$, Disease Free Equilibrium is stable when $\tau=0.67$. This stability is only achieved if drug efficacy is administered. The results show that when drug efficacy of $\alpha_{1}=0.723$ and $\alpha_{2}=0.723$ the DFE is achieved.
\end{abstract}

Keywords: HIV, Reproduction Number, Delay, Stability, Disease Free Equilibrium, Chemotherapy

\section{Introduction}

Human immunological disorder Virus (HIV) is one of the transmittable diseases thought to significantly cause death in developing countries like Kenya. HIV is categorized into two namely; HIV-1 and HIV-2. This paper will only focus on HIV-1 which is often chargeable for the bulk of HIV contagions worldwide. Radical point of HIV contagion is Acquired Immuno Deficiency virus (AIDs) which is contracted over direct contact with HIV-disease-ridden body fluids that include blood, semen, and reproductive organ secretions, or from HIV-infected mother to child through gestation, delivery or breastfeeding $[10,12]$. T-helper is a type of the white blood cell that is targeted mainly by the HIV as soon as it enters the human body. Once it binds itself to this cell, the HIV life cycle immediately begins. HIV viral replication is rampant in the first stage of contagion [14]. Many scholars in the field of biological mathematics have designed models that explain the HIV-1 in vivo dynamics [1, 7, 11, 18]. A new HIV viral material submits to seven steps namely; binding, fusion, reverse transcription, integration, replication, assembly and budding [2-3, 8-10, 17]. The basic reproduction number $R_{0}$ is the most substantial measure in contagious disease epidemiology such as HIV. This parameter is one of the measures projected for emergent contagious diseases in outbreak state of affairs and its significance provide discernment when designing control 
interventions for established contagions. $R_{0}$ is the typical amount of new cases of contagion caused by one distinctive disease-ridden person in a population comprising of vulnerable only. $R_{O}$, forms a threshold value for most models of infectious diseases since for $R_{O}<1$, the disease-free equilibrium point is stable $[5,13,15]$. Various mathematical models have been developed to explain internal viral dynamics of HIV. In [6] intracellular delay in virus production at the side of the delay in immunologic response is studied where it is shown that the contagion free balanced state is steady when $R_{o}<1$. This means that the contagion are often cleared if ARVs medical care is administered. In [4] a mathematical model that studies underlying transmission mechanisms of HIV infections is established and it is shown that when $R_{0}>1$ the disease free equilibrium state is asymptotically unstable and hence unwellness persist. In [7] a model that looks into the consequences of time delay on HIV undercurrents in the existence of ARVs ill-treatment delay differential equations is considered where it is shown that CD4+T-cells stay inactive for a short time and the HIV infective agent materials do not seem to be reproduced and that the system at the side of treatment gets enough time to clear the infective agent material within the blood hence maintaining the Endemic Equilibrium Point. However, in their study the used and unused CD8+T-cells were not considered. In [10] in-vivo dynamics of HIV subjected to the influence of CD8+T-cells is considered. It is proved that the virions free equilibrium state is domestically stable if $\mathrm{Ro}<1$ and unstable otherwise. In their study however, they did not take into account effects of time delay on HIV-1 dynamics.

In this paper, we have formulated deterministic mathematical Susceptible Infectious Recovered (SIR) models has described the HIV-1 in vivo dynamics in presence of chemotherapy using delay differential equations. The model has six variables: Healthy CD4+T-cells $(u)$, Sick CD4+Tcells $(v)$, Infectious virus $(w)$, Non-infectious virus $(x)$, used CD8+T-cells $(y)$ and un-used CD8+T-cells $(z)$.

\section{Methods of Solution}

The stability of the model has been approached from Jacobian matrix method of checking stability of Disease Free

$$
(u(0)>0, v(0)>0, w(0)>0, x(0)>0, y(0)>0, z(0)>0) \in C[7,10]
$$

In addition, we assume that the initial data for the system (1) satisfies

$$
u_{0}(\psi) \geq 0, v_{0}(\psi) \geq 0, w_{0}(\psi) \geq 0, x_{0}(\psi) \geq 0, y_{0}(\psi) \geq 0, z_{0}(\psi) \geq 0, \psi \in[-\tau, 0]
$$

The following theorem establishes the positivity and boundedness of solution with initial functions satisfying (2) and (3)

Theorem 2.1.2 let $(u, v, w, x, y, z)$ be the solution of system (1) satisfying conditions (2) and (3) then $u, v, w, x, y$

$$
\begin{gathered}
u(t)=u(0) e^{-\int_{0}^{t} \varepsilon_{u}+\left(1-\alpha_{1}\right) \omega(\xi-\tau) d \xi}+\int_{0}^{t}\left[\alpha_{1} v(\eta)+\psi\right] e^{-\int_{\eta}^{t} \varepsilon_{u}+\left(1-\alpha_{1}\right) \omega w(\xi-\tau) d \xi} d \eta \\
v(t)=v(0) e^{-\int_{0}^{t}\left(\varepsilon_{v}+\alpha_{1}-\varphi v_{\tau} y\right) d \xi}+\int_{0}^{t}\left[\left(1-\alpha_{1}\right) \omega w(\eta-\tau) u(\eta)\right] e^{\left.\left.-\int_{\eta}^{t} \varepsilon_{v}+\alpha_{1}-\varphi_{1} v_{\tau} y\right)\right) d \xi} d \eta
\end{gathered}
$$




$$
\begin{gathered}
w(t)=w(0) e^{-\int_{0}^{t} \varepsilon_{w} d \xi}+\int_{0}^{t}\left[\left(1-\alpha_{1}\right)\left(1-\alpha_{2}\right) p v(\eta-\tau)\right] e^{-\int_{\eta}^{t} \varepsilon_{w} d \xi} d \eta \\
x(t)=x(0) e^{-\int_{0}^{t} \varepsilon_{x} d \xi}+\int_{0}^{t}\left[\left(1-\alpha_{1}\right) \alpha_{2} p v(\eta-\tau)\right] e^{-\int_{\eta}^{t} \varepsilon_{x} d \xi} d \eta \\
y(t)=y(0) e^{\left.-\int_{0}^{t}\left(\varphi v_{\tau}-\varepsilon_{y}\right)\right) d \xi} \\
z(t)=z(0) e^{-\int_{0}^{t}\left(-\varepsilon_{z}\right) d \xi}
\end{gathered}
$$

Positivity immediately follows from (2) and (3)

For boundedness we define:

$$
N(t)=u(t)+v(t)+w(t)-\frac{1-\alpha_{2}}{\alpha_{2}} x(t)+y(t)+z(t)
$$

Let $\pi=\min \left(\varepsilon_{u} ; \varepsilon_{z} ; \varepsilon_{y} ; \varepsilon_{v} ; \varepsilon_{w} ; \varepsilon_{x}\left(\frac{1-\alpha_{2}}{\alpha_{2}}\right)\right)$ then:

$$
\frac{d}{d t} N(t)=\frac{d}{d t} u(t)+\frac{d}{d t} v(t)+\frac{d}{d t} w(t)-\frac{1-\alpha_{2}}{\alpha_{2}} \frac{d}{d t} x(t)+\frac{d}{d t} y(t)+\frac{d}{d t} z(t)
$$

Thus:

$$
\frac{d}{d t} N(t) \leq \omega-\pi N(t) \text { where } \omega=\psi+\sigma
$$

Implying that $N(t)$ is bounded, and so are $u(t), v(t), w(t), x(t), y(t), z(t)$. This completes the proof.

\subsection{Computation of Basic Reproduction Number $R_{0}$}

In this paper, we adopt next generation matrix approach in computation of $R_{0}$, such that $R_{0}=\rho\left(F V^{-1}\right)$ where $\rho$ is the spectral radius of next generation matrix, while $F$ is the matrix of the infection and $V$ is the matrix of transfer of individuals out of the compartment by other means. To obtain $R_{0}$ the dominant eigenvalue of the NGM is considered $[9,16$, 18]. In system (1), there are two infection classes, hence, at disease free equilibrium the matrix of new infection is given by; matrix $F$ and $V$

Matrix $F_{i}$ obtained by considering the rate of new infections entering compartment $i$,

$$
F_{i}=\left[\begin{array}{c}
0 \\
\left(1-\alpha_{1}\right) \omega w_{\tau} u \\
\left(1-\alpha_{1}\right)\left(1-\alpha_{2}\right) p v_{\tau} \\
0 \\
0 \\
0
\end{array}\right]
$$

The matrix that represents the rate of transfer into and out of compartment $\mathrm{i}$ by any other means at DFE is given by matrix V;

$$
V_{i}=\left[\begin{array}{c}
0 \\
\left(\varepsilon_{v}+\gamma \alpha_{1}+z\right) \varphi v \\
\varepsilon_{w} w \\
0 \\
0 \\
0
\end{array}\right]
$$

Clearly,

$$
\begin{gathered}
F=\left[\begin{array}{cc}
0 & \left(1-\alpha_{1}\right) e^{-\lambda \tau} \frac{\psi}{\varepsilon_{u}} \\
\left(1-\alpha_{1}\right)\left(1-\alpha_{2}\right) p e^{-\lambda \tau} & 0
\end{array}\right] \\
V=\left[\begin{array}{cc}
\left(\varepsilon_{v}+\alpha_{1}+\varphi \frac{\sigma}{\varepsilon_{z}} e^{-\lambda \tau}\right. & 0 \\
0 & \varepsilon_{w}
\end{array}\right]
\end{gathered}
$$

Then, the inverse of $V$ is given by;

$$
V^{-1}=\left[\begin{array}{cc}
\frac{1}{\left(\varepsilon_{v}+\alpha_{1}+\varphi \frac{\sigma}{\varepsilon_{z}} e^{-\lambda \tau}\right.} & \frac{1}{\varepsilon_{w}}
\end{array}\right]
$$

The next generation matrix $F V^{-1}$ is given by:

$$
F V^{-1}=\left[\begin{array}{cc}
0 & \left(1-\alpha_{1}\right) e^{-\lambda \tau} \psi \\
\frac{\varepsilon_{u} \varepsilon_{w}}{\left(\varepsilon_{v}+\alpha_{1}+\varphi \frac{\sigma}{\varepsilon_{Z}} e^{-\lambda \tau}\right.} & 0
\end{array}\right]
$$

The eigenvalues of the matrix (16) can be obtained as;

$$
\left[\begin{array}{cc}
0-\lambda^{*} & \left(1-\alpha_{1}\right)\left(1-\alpha_{2}\right) p e^{-\lambda \tau} \\
\frac{\left(1-\alpha_{1} \psi\right.}{\left(\varepsilon_{v}+\alpha_{1}+\varphi \frac{\sigma}{\varepsilon_{z}} e^{-\lambda \tau}\right)} & \frac{\left(1 \varepsilon_{w}\right.}{0-\lambda^{*}}
\end{array}\right]
$$

Characteristic equation is of the above matrix is given by;

$$
\lambda^{* 2}-\left(\frac{\left(1-\alpha_{1}\right)\left(1-\alpha_{2}\right) p e^{-\lambda \tau}}{\left(\varepsilon_{v}+\alpha_{1}+\varphi \frac{\sigma}{\varepsilon_{z}} e^{-\lambda \tau}\right)}\right)\left(\frac{\left(1-\alpha_{1}\right) e^{-\lambda \tau} \psi}{\varepsilon_{u} \varepsilon_{w}}\right)=0
$$

whose roots are:

$$
\begin{gathered}
\lambda_{1,2}^{*}= \pm \sqrt{\frac{\left(1-\alpha_{1}\right)^{2}\left(1-\alpha_{2}\right) p e^{-2 \lambda \tau}}{\left(\varepsilon_{v}+\alpha_{1}+\varphi \frac{\sigma}{\varepsilon_{z}} e^{-\lambda \tau}\right)\left(\frac{\psi}{\varepsilon_{u} \varepsilon_{W}}\right)}} \\
\lambda^{*}{ }_{1}=\sqrt{\left.\frac{\left(1-\alpha_{1}\right)^{2}\left(1-\alpha_{2}\right) p e^{-2 \lambda \tau}}{\left(\varepsilon_{v}+\alpha_{1}+\varphi \frac{\sigma}{\varepsilon_{z}} e^{-\lambda \tau}\right)}\right)\left(\frac{\psi}{\varepsilon_{u} \varepsilon_{W}}\right)}
\end{gathered}
$$




$$
\lambda_{2}^{*}=-\sqrt{\left.\frac{\left(1-\alpha_{1}\right)^{2}\left(1-\alpha_{2}\right) p e^{-2 \lambda \tau}}{\left(\varepsilon_{v}+\alpha_{1}+\varphi \frac{\sigma}{\varepsilon_{z}} e^{-\lambda \tau}\right)}\right)\left(\frac{\psi}{\varepsilon_{u} \varepsilon_{W}}\right)}
$$

Thus the $R_{0}$ which is given by the greatest eigenvalue is:

$$
R_{0}=\sqrt{\left.\frac{\left(1-\alpha_{1}\right)^{2}\left(1-\alpha_{2}\right) p e^{-2 \lambda \tau}}{\left(\varepsilon_{v}+\alpha_{1}+\varphi \frac{\sigma}{\varepsilon_{Z}} e^{-\lambda \tau}\right)}\right)\left(\frac{\psi}{\varepsilon_{u} \varepsilon_{w}}\right)}
$$

\section{Remark 2.1.3}

Equilibrium Points and Their Stability

Here, we study the stability of model (1). In mathematical epidemiology we usually consider two equilibria points that is, Disease Free Equilibrium Point (DFE) and the Endemic Equilibrium Point (EEP). We analyze the stability of system (1) by estimating the $R_{0}$ using Next Generation Matrix approach and then check its stability using Jacobian matrix.

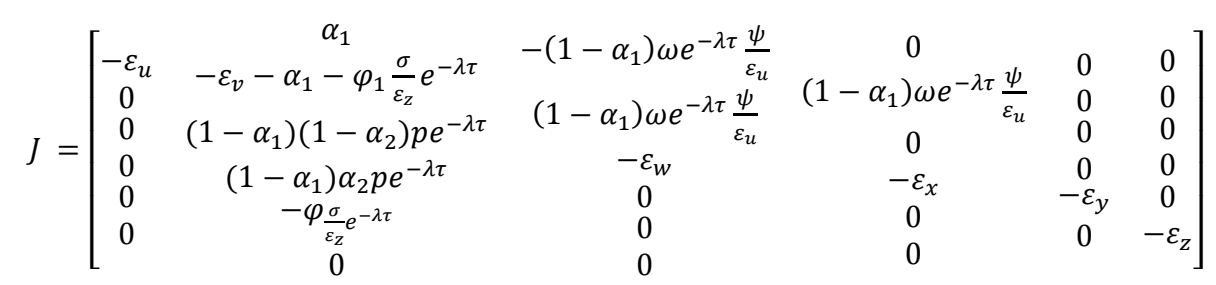

The system (1) is locally asymptotically stable if all the eigenvalues of linearization matrix of system (1) are negative. Clearly, the eigenvalues of (21) are:

$$
\begin{gathered}
\lambda_{1}^{*}=-\varepsilon_{u}, \lambda^{*}{ }_{2}=-\varepsilon_{x}, \lambda^{*}{ }_{3}=-\varepsilon_{y}, \lambda^{*}{ }_{4}=-\varepsilon_{z} \\
\lambda^{*}{ }_{5,6}=\frac{-\varepsilon_{w}\left(\varepsilon_{v}+\alpha_{1}+\varphi \frac{\sigma}{\varepsilon_{z}} e^{-\lambda \tau}\right) \pm \sqrt{\left(\varepsilon_{w}\left(\varepsilon_{v}+\alpha_{1}+\varphi \frac{\sigma}{\varepsilon_{z}} e^{-\lambda \tau}\right)\right)^{2}-4\left(\left(\varepsilon_{w}\left(\varepsilon_{v}+\alpha_{1}+\varphi \frac{\sigma}{\varepsilon_{z}} e^{-\lambda \tau}\right)\right)-\left(\frac{\left(1-\alpha_{1}\right)^{2}\left(1-\alpha_{2}\right) \omega p e^{-2 \lambda \tau} \psi}{\varepsilon_{u}}\right)\right)}}{2}
\end{gathered}
$$

From (22) it is evident that $\lambda^{*}{ }_{1}, \lambda^{*}{ }_{2}, \lambda^{*}{ }_{3}, \lambda^{*}{ }_{4}, \lambda^{*}{ }_{5}$ are all negative. The stability of our model will be determined by the sign of $\lambda^{*}{ }_{6}$

$$
\begin{array}{r}
\lambda^{*}{ }_{6}=-\varepsilon_{w}\left(\varepsilon_{v}+\alpha_{1}+\varphi \frac{\sigma}{\varepsilon_{z}} e^{-\lambda \tau}\right)+\sqrt{\left(\varepsilon_{w}\left(\varepsilon_{v}+\alpha_{1}+\varphi \frac{\sigma}{\varepsilon_{z}} e^{-\lambda \tau}\right)\right)^{2}-4\left(\left(\varepsilon_{w}\left(\varepsilon_{v}+\alpha_{1}+\varphi \frac{\sigma}{\varepsilon_{z}} e^{-\lambda \tau}\right)\right)-\left(\frac{\left(1-\alpha_{1}\right)^{2}\left(1-\alpha_{2}\right) \omega p e^{-2 \lambda \tau} \psi}{\varepsilon_{u}}\right)\right)<0}(23) \\
\text { If }-\varepsilon_{w}\left(\varepsilon_{v}+\alpha_{1}+\varphi \frac{\sigma}{\varepsilon_{z}} e^{-\lambda \tau}\right)>\sqrt{\left(\varepsilon_{w}\left(\varepsilon_{v}+\alpha_{1}+\varphi \frac{\sigma}{\varepsilon_{z}} e^{-\lambda \tau}\right)\right)^{2}-4\left(\left(\varepsilon_{w}\left(\varepsilon_{v}+\alpha_{1}+\varphi \frac{\sigma}{\varepsilon_{z}} e^{-\lambda \tau}\right)\right)-\left(\frac{\left(1-\alpha_{1}\right)^{2}\left(1-\alpha_{2}\right) \omega p e^{-2 \lambda \tau} \psi}{\varepsilon_{u}}\right)\right)}
\end{array}
$$

then it follows that;

$$
\left.\frac{\left(1-\alpha_{1}\right)^{2}\left(1-\alpha_{2}\right) p e^{-2 \lambda \tau}}{\left(\varepsilon_{v}+\alpha_{1}+\varphi_{1} \frac{\sigma}{\varepsilon_{Z}} e^{-\lambda \tau}\right.}\right)\left(\frac{\psi}{\varepsilon_{u} \varepsilon_{w}}\right)<1 \text { or } \sqrt{\left.\frac{\left(1-\alpha_{1}\right)^{2}\left(1-\alpha_{2}\right) p e^{-2 \lambda \tau}}{\left(\varepsilon_{v}+\alpha_{1}+\varphi \frac{\sigma}{\varepsilon_{Z}} e^{-\lambda \tau}\right)}\right)\left(\frac{\psi}{\varepsilon_{u} \varepsilon_{w}}\right)}<1
$$

Therefore, $R_{0}<1$ is attained for DFE to be stable.

\section{Main Results}

Analytic solutions can be demonstrated using analytic results with specific numerical examples. The model equation (1) is considered. Numerical simulations of the model is calculated using list of parameters and their estimated values given in the table $1 \& 2$. The values have been obtained from $[1,7,10]$. In simulation of the model system (1), the following initial values in each compartment at the onset of infection is assumed to apply; $u(0), v(0), w(0), x(0), y(0), z(0)=(1000,0,0.01,0.01,500,30)$ on the interval $[-\tau, 0]$.

Table 1. Table of Variable, Variable description and Value.

\begin{tabular}{lll}
\hline Variables & Variable description & Value \\
\hline$u$ & Healthy CD4+ T- cells & 1000 \\
$v$ & Sick CD4+ T- cells & 0 \\
$w$ & Infectious virus & 0.001 \\
$x$ & Non-infectious virus & 0.001 \\
$y$ & CD8+ T-cells used & 500 \\
$z$ & CD8+ T-cells un-used & 30 \\
\hline
\end{tabular}


Table 2. Table of Parameters, Parameter description and Value.

\begin{tabular}{|c|c|c|}
\hline Parameters & Parameter description & Value \\
\hline$\psi$ & $\begin{array}{l}\text { Production rate of healthy } \mathrm{CD}^{+} \mathrm{T} \\
\text { cells }(u)\end{array}$ & $\begin{array}{l}15 \\
\text { cells } / \mathrm{mm}^{3} / \text { day }\end{array}$ \\
\hline$\varepsilon_{u}$ & Death rate of healthy $\mathrm{CD} 4^{+} \mathrm{T}$ cells $(u)$ & $0.06 /$ day \\
\hline$\alpha_{1}$ & Efficacy of RTI & $0 \leq \alpha_{1} \leq 1$ \\
\hline$\alpha_{2}$ & Efficacy of PI & $0 \leq \alpha_{2} \leq 1$ \\
\hline$\omega$ & Force of infection & 0.53 \\
\hline$p$ & $\begin{array}{l}\text { Budding size of free virus from sick } \\
\mathrm{CD} 4^{+} \mathrm{T} \text { cells }\end{array}$ & 13 \\
\hline$\varepsilon_{v}$ & Death rate of sick $\mathrm{CD} 4^{+} \mathrm{T}$ cells & 0.26 \\
\hline$\varepsilon_{w}$ & $\begin{array}{l}\text { Natural death rate of infectious virus } \\
\text { from the body }\end{array}$ & 2.4 \\
\hline$\varepsilon_{x}$ & $\begin{array}{l}\text { Natural death rate of non-infectious } \\
\text { virus from the body }\end{array}$ & 2.4 \\
\hline$\varepsilon_{y}$ & $\begin{array}{l}\text { Natural death rate of used CD8+ T- } \\
\text { cells }\end{array}$ & $0.06 /$ day \\
\hline$\varepsilon_{z}$ & $\begin{array}{l}\text { Natural death rate of un-used CD8+ T- } \\
\text { cells }\end{array}$ & $0.06 /$ day \\
\hline$\sigma$ & Production rate of CD8+ T-cells & $0.004 /$ day \\
\hline$\tau$ & Time delay & $\begin{array}{l}\text { To be } \\
\text { determined }\end{array}$ \\
\hline$\varphi$ & Rate at which CD8+ T-cells are used & $20 \operatorname{cell} / \mathrm{mm}^{3} /$ day \\
\hline
\end{tabular}

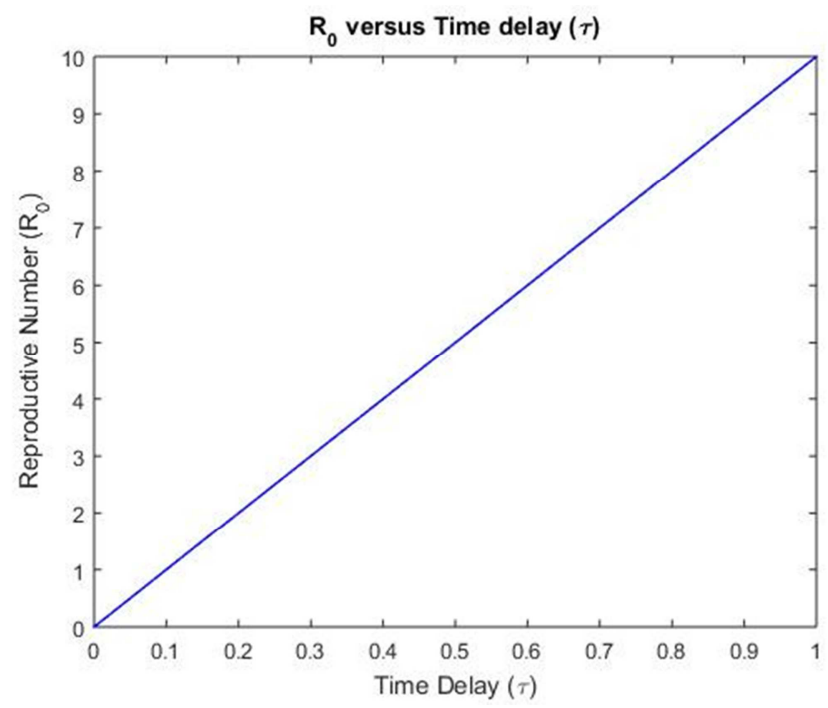

Figure 1. Figure showing $R_{0}$ with respect to Time Delay.

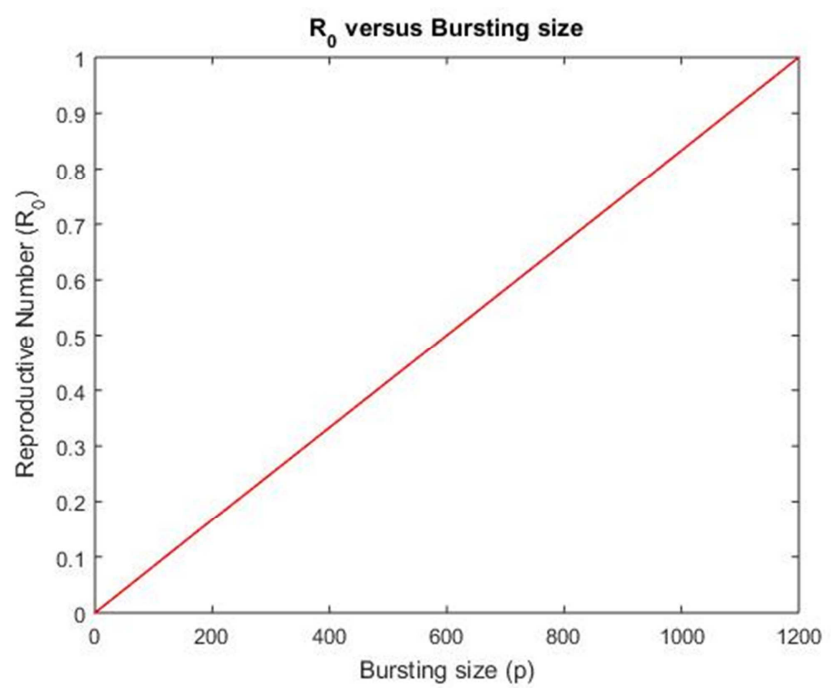

Figure 2. A figure showing $R_{0}$ with respect to bursting size.

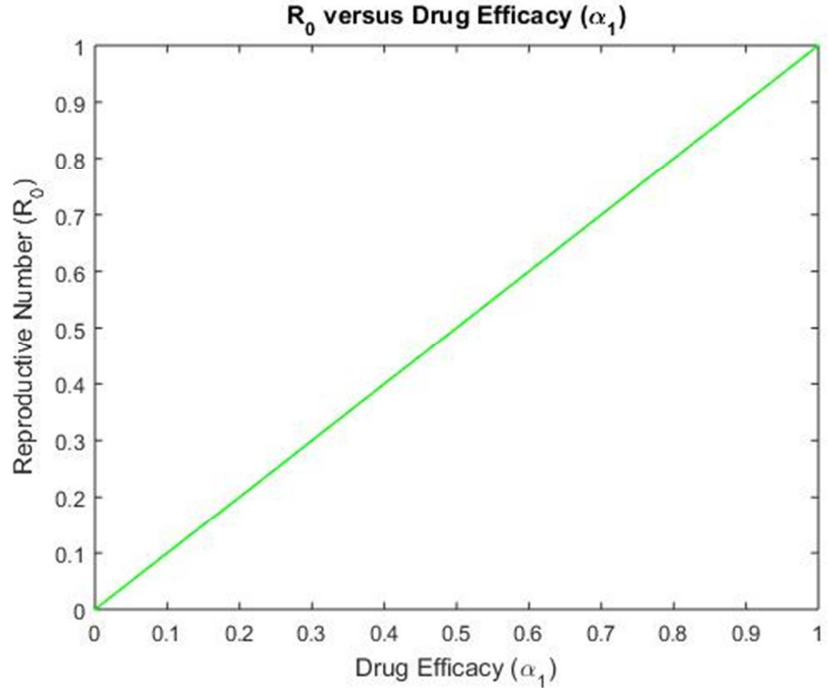

Figure 3. Figure showing $R_{0}$ with respect to Drug Efficacy.

Figure 1 A figure showing Reproduction number with respect to time delay. It is evident that if time delay is large viral replication is high. It is clear that $R_{0}<1$ for $0<\tau<1$. Therefore Disease Free Equilibrium is stable for $\tau<1$.

Figure 2 A figure showing Reproduction number with respect to bursting size. It is evident that $R_{0}$ is directly proportional to the bursting size. It is clear that $R_{0}<1$ if $0<p<200$. For DFE to be stable $p<200$.

Figure 3 A figure showing Reproduction number with respect to drug efficacy at $80 \%$ efficacy. It is evident that DFE is achieved when drug efficacy is at $80 \%$. Therefore, chemotherapy played a major role in reducing the viral replication for stability to be achieved at $R_{0}<1$.

Figure 4 A figure showing Reproduction number with respect to immune response (CD8+T-cells). It is evident that immune response plays a role in reducing viral replication. Clearly, for $R_{0} \leq 1$ for CD8+ $\mathrm{T}$ cells $\leq 30$. Stability of Disease Free Equilibrium is achievable in the presence of CD8+ T cells.

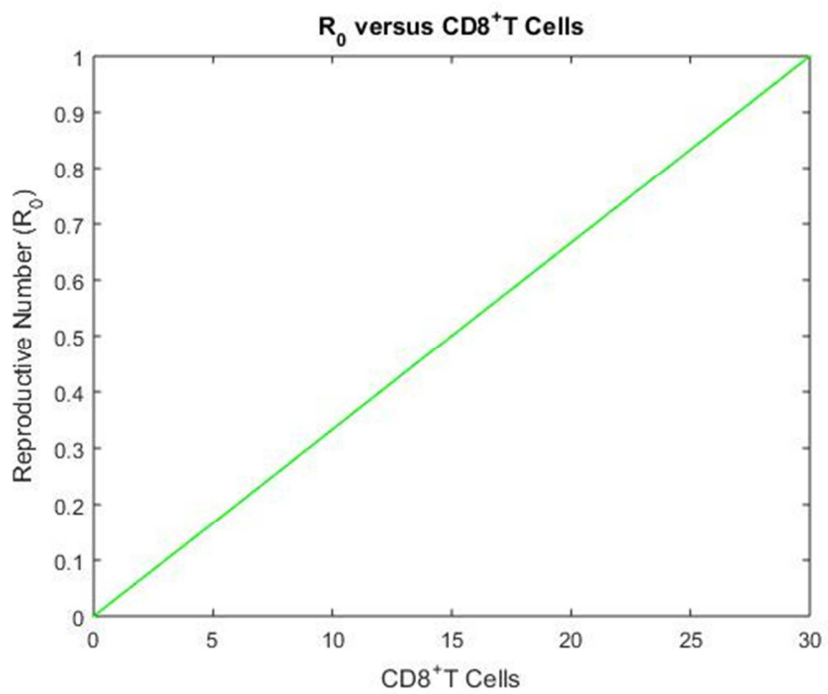

Figure 4. Figure showing $R_{0}$ with respect to $C D 8+T$-cells. 
Figure 5 A figure showing the dynamics population of CD4+T-cells and contagious viral cells with neither treatment nor immune response. It is evident that initially the contagious virus was at zero and immediately its number shots to $4000 / \mathrm{mm}^{3}$ due to rapid viral replication. As a result CD4+T-cells count goes down.

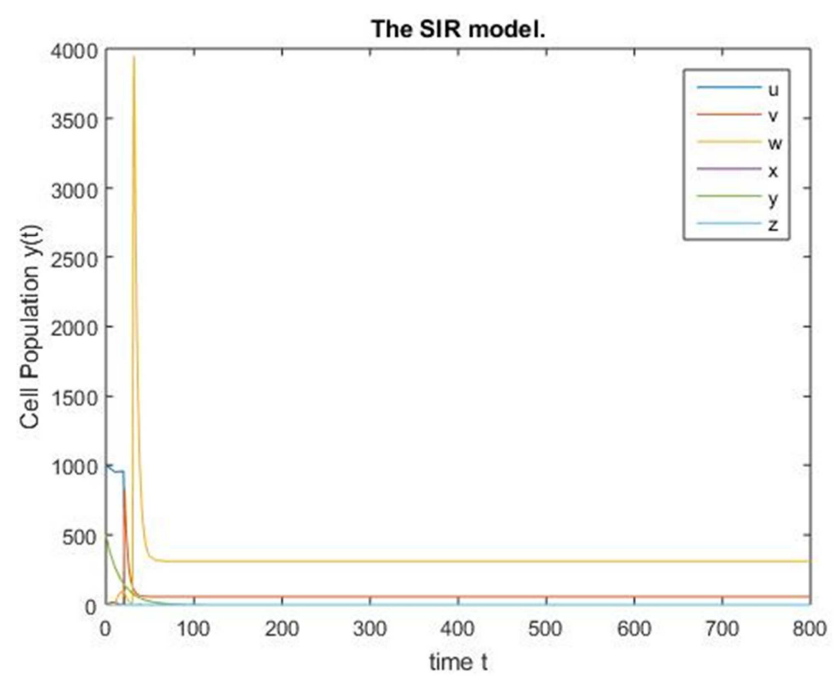

Figure 5. Dynamics population of CD4+T-cells and contagious cells with neither treatment nor immune response.

Figure 6 A figure showing the dynamics population of CD4+T-cells with $20 \%$ drug efficacy and immune response. It is evident that CD4+ T-cells count is at $2500 / \mathrm{mm}^{3}$ after some time which is a value lower than $4000 / \mathrm{mm}^{3}$ that was evident when there were no treatment and immune response. This implies that chemotherapy and immune response played a major role in reducing the replication of the contagious virus and delaying it to happen.

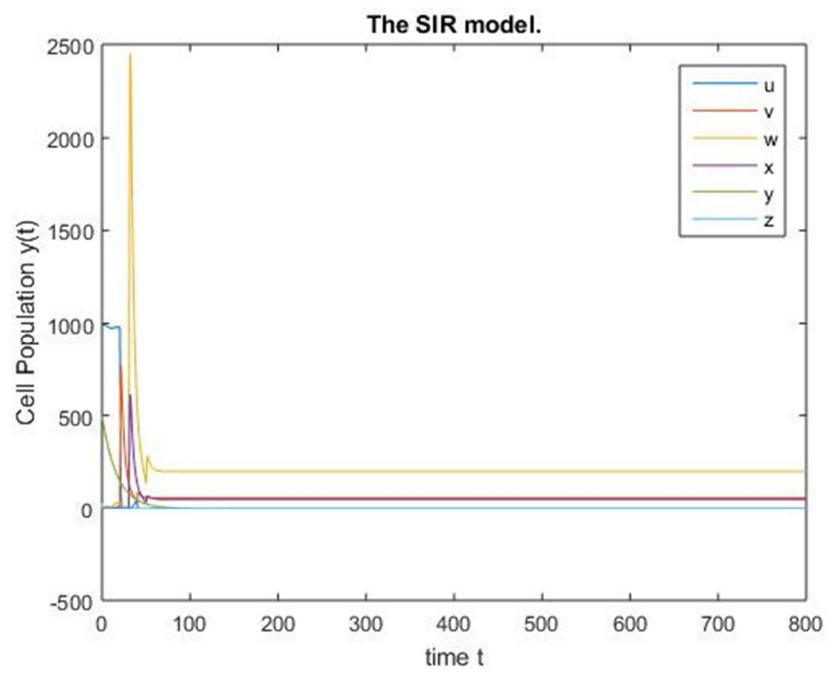

Figure 6. Dynamics population of CD4+T-cells and contagious cells with $20 \%$ drug efficacy and immune response with respect to time.

Figure 7 A figure showing dynamic population of CD4+Tcells and contagious viral cells with $80 \%$ drug efficacy and immune response. It is evident that contagious viral replication rises slowly to a value slightly above $200 / \mathrm{mm}^{3}$.
The CD4+T-cells are maintained at a value above 200 cell $/ \mathrm{mm}^{3}$.

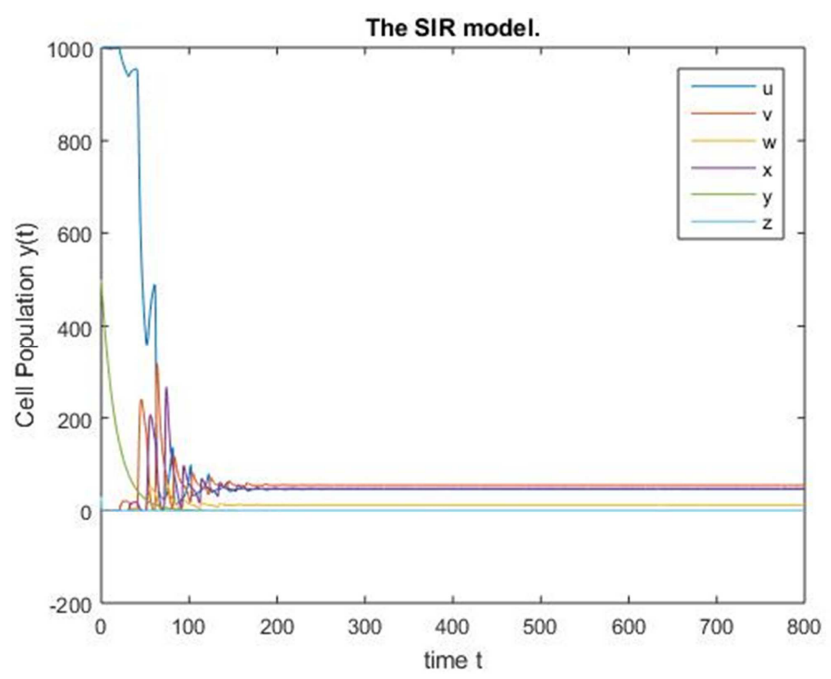

Figure 7. Dynamics population of CD4+T-cells and contagious cells with $80 \%$ drug efficacy and immune response with respect to time.

\section{Conclusion}

In this paper, the main objective is to study the dynamics of HIV-1 in-vivo in the presence of time delay, chemotherapy and role of $\mathrm{CD} 8+\mathrm{T}$-cells. Disease Free Equilibrium is attained when $R_{0}<1$. This reproduction number has been obtained using NGM and Jacobian matrix and it is clearly seen that $R_{0}$ is affected by delay, immune response and chemotherapy of both RTI and PI. The study found out that if all other factors are kept constant then DFE is achieved when drug efficacies for RTI and PI are at 0.723 . Any values of efficacies above this value has no change in dynamics whereas any value below this may be as good in decreasing the reproduction number. It is also clear that for $\tau>0$ DFE is stable and unstable otherwise. Time delay, immune response and chemotherapy plays an important role in reducing the replication of HIV virus and stability at DFE. Thus elimination of HIV contagious virus are achieved through use of chemotherapy, time delay and CD8+T-cells from the contagion (entering of virus into the cell) to bursting of the contagious virus. Prolonging this time, then the drop in CD4+T-cells count will not deplete within a shorter period.

\section{Suggestions for Further Research}

This study has not explored all about HIV-1 In vivo dynamics. Sensitivity analysis and Hopf bifurcation is not captured in the study. Also, Studies on chemotherapy using Structured Treatment Interference (STI) regime by narrowing to the delay for stabilities at EEP is not captured.

\section{References}

[1] Arruda, E. D. (2015). An Optimal Control Approach to HIV Immunology. Applied Mathematics, 1115-1130. 
[2] Bonhoeffer, R. M. (2013). Production of resistant HIV mutants during antiretroviral therapy. Journal of Apllied Mathematics.

[3] Fisher, K. A. (2013). Stability conditions of HIV co-infection model using Next generation method. Journal of Applied Mathematics Science, 2815-2832.

[4] Hatta, K. A. (2012). Optimal control of a Delayed HIV infection model with immune Response using an efficient Numerical method. Journal of ISRN Biomathematics, 1-7.

[5] Jinliang Wang, J. (2015). Analysis of an age structured HIV infection model with virus-to-cell infection and cell-to-cell transmission. Journal of Apllied Mathematics.

[6] Kasia A Pawelek, S. L. (2012). A model of HIV-1 infection with two time delays: Mathematics analysis and comparison with patient data. Journal of Mathematics Biology, 98-109.

[7] Kirui Wesley, R. K. (2015). Modelling the effects of time delay on HIV-1 in vivo dynamics in the presence of ARVs. Science Journal of Applied Mathematics and Statistics, 204213.

[8] Michael Y Li, H. (2012). Global dynamics of a mathematical model for HTLV-1 infection of CD4+ T cells with delayed with CTL response. Journal of Mathematics Biology, 10801092.

[9] Nakul, C. (2011). The basic reproduction number. Swiss: Swiss Tropical and Public Health Insitute.

[10] Ngina Purity M., R. W. (2017). Mathematical modelling of in vivo dynamics of HIV subject to the influence of the CD8+ Tcells. Journal of Applied Mathematics, 1153-1179.
[11] Ngina Purity M., R. W., L. S. L. (2018). Modelling Optimal Control of In-Host Dynamics Using Different Control Strategies. Journal of Applied Mathematics. https://doi.org/10.1155/2018/9385080.

[12] Perelson, S. (1989). modelling the interaction of immune system with HIV in mathematical and stastical approaches to AIS epidemiology. Journal of Mathematical and Statistics Appraoches on AIDs Epidemiology, 350-370.

[13] Picker, A. (2013). CD4+ T-cells depletion in HIV infection. Journal of Mathematical Biology, 54-64.

[14] Prashant K. Srivastava, M. B. (2012). Dynamic model of inhost HIV infection with drug therapy and multiviral strains. Journal of Biology Systems, 303-325.

[15] Salantes, D. B. (2018). Rebound Relationships. An Investigation of HIV-1 Rebound Dynamics and Host Immune Response During Analytical Treatment Interruption. Publicly Accessible Penn Dissertations. 3179.

[16] Assone T., A. (2016). Genetic makers of the host in persons living with HTLV-1, HIV and HCV infections. Journal of Mathematical Biology.

[17] Tianlei Wang, Z. H. (2015). Global stability analysis for delayed virus infection model with general incidence rate and humoral immunity. Journal of Mathematics and computers in simulation $89,13-22$.

[18] Waema. R. M., L. L. (2013). Stochastic model for in-host HIV dynamics with therapeutic intervention. Journal of ISRN Biomathematics, 11 pages. 